\title{
Originalartikel
}

\section{Toxizität von Thymol, Campher, Menthol und Eucalyptol auf Varroa jacobsoni Oud und Apis mellifera L im Labortest}

\author{
A Imdorf, V Kilchenmann, S Bogdanov, B Bachofen, C Beretta
}

Forschungsanstalt für Milchwirtschaft, Sektion Bienen, CH-3097 Liebefeld, Schweiz

(Eingegangen 15 Juni 1994; angenommen 29 September 1994)

\begin{abstract}
Zusammenfassung - Zur Ermittlung der Toxizität von ätherischen Substanzen auf Varroa jacobsoni und Apis mellifera wurde ein Labortest entwickelt. Dabei wurden gekäfigte Bienen und Varroa in Exsikkatoren während 72 Stunden unterschiedlichen Konzentrationen von Thymol, Campher, Menthol und Eucalyptol in der Luft ausgesetzt. Die Konzentrationen, bei denen nahezu $100 \%$ der Varroa getötet wurden und die Bienen ohne nennenswerte Verluste überlebten, lagen für Thymol zwischen 5 und 15 $\mu \mathrm{g} / \mathrm{l}$, für Campher zwischen 50 und $150 \mu \mathrm{g} / \mathrm{l}$ und für Menthol zwischen 20 und $60 \mu \mathrm{g} / \mathrm{l}$ Luft. Bei Eucalyptol hingegen wurde bei einer Varroa-Mortalität von $100 \%$ bereits eine Bienenmortalität von $25 \%$ festgestellt.
\end{abstract}

Apis mellifera / Varroa jacobsoni / Toxizität / ätherische Substanz / chemische Bekämpfung

\section{EINLEITUNG}

Hoppe (1990) überprüfte 55 verschiedene ätherische Öle auf ihre Toxizität bei Varroa und Bienen nach topikaler Applikation und indem die Tiere den Dämpfen dieser Öle ausgesetzt wurden. Dabei zeigten nur Nelken- und das Wintergrünöl sowohl eine gute varroazide Wirkung als auch eine gute Bienenverträglichkeit.

Nach verschiedenen Autoren (Chiesa, 1991, Literaturübersicht) hat Thymol bei geeigneter Anwendung eine gute varroazide Wirkung. Das Produkt 'Apilife VAR', welches neben Thymol (76\%) kleinere Men- gen von Eucalyptol, Campher und Menthol enthält, weist bei der Varroa-Bekämpfung im Schweizerkasten mit $97 \%$ Behandlungserfolg eine sehr gute Wirksamkeit auf (Rickli et al, 1991; Imdorf et al, 1994). In verschiedenen Magazinbeuten muss vor allem in Völkern mit zwei Zargen mit einem geringeren Behandlungserfolg gerechnet werden (Van der Steen, 1992; Liebig, 1993; Mutinelli et al, 1993; Schulz, 1993; Vorwohl et al, 1994). Der geringe Anteil von Campher in diesem Produkt hat keinen Einfluss auf den Behandlungserfolg (Imdorf et al, 1994). Inwiefern Eucalyptol und Menthol zur Wirksamkeit beitragen, ist nicht bekannt. Eine zu hohe Thymolkonzentration in der 
Stockluft kann zu Brut- und Bienenverlusten führen (Mautz, 1982; Liebig, 1993; Moosbeckhofer, 1993). Menthol reduziert den Befallsgrad von Acarapis woodi bei Bienen je nach Dosierung und Anwendungszeitpunkt unterschiedlich stark (Vecchi und Giordani, 1968; Cox et al, 1986, 1989; Wilson et al, 1990; Duff und Furgula, 1991, 1993; Delaplane, 1992).

Um eine optimale Wirkung bei der Varroabekämpfung erreichen zu können, ist es notwendig, jenen Konzentrationsbereich in der Luft zu kennen, bei dem die Varroa getötet, die Bienen jedoch nicht geschädigt werden. In der vorliegenden Studie wurde ein Labortest entwickelt und die Dosis-WirkungsBeziehung von 4 ätherischen Substanzen auf Varroa-Milben und Bienen ermittelt.

\section{MATERIAL UND METHODEN}

Zwei Liebefelder Käfige mit seitlichen Gitterabdeckungen, welche je ca 100 Bienen mit 20 bis 40 Varroa enthielten, wurden bei $32^{\circ} \mathrm{C}$ in einen Exsikkator gestellt. Die Bienen stammten aus brutlosen Völkern mit eingesperrten Königinnen. Sie wurden mit sterilisiertem Zuckerwasser (1:1) gefüttert. Die Exsikkatoren wurden während 72 Stunden mit einem Gemisch aus Frischluft (ca $500 \mathrm{ml} / \mathrm{min}$ ) und mit Thymol, Eucalyptol, Campher oder Menthol angereicherter Luft (5-130 $\mathrm{ml} / \mathrm{min}$ ) versorgt. Die Herstellung der kontaminierten Luft erfolgte, indem frische Luft durch eine Gaswaschflasche $(250 \mathrm{ml})$, welche $20 \mathrm{~g}$ des Wirkstoffs enthielt, geleitet wurde. Ein Exsikkator diente bei jedem Versuch als Kontrolle und wurde nur mit Frischluft (ca $550 \mathrm{ml} / \mathrm{min}$ ) beschickt. Nach 24, 48 und 72 Stunden wurde in jedem Exsikkator eine Luftprobe (1-20 I, je nach Wirkstoff und Konzentration) entnommen. Dabei wurde die Luft mit einer Pumpe (je nach Konzentration des Wirkstoffes bis $300 \mathrm{ml} / \mathrm{min}$ ) durch ein Absorptionsröhrchen (Orbo-101, Supelco) angesogen. Die Wirkstoffe wurden anschliessend in Toluol extrahiert und gaschromatographisch analysiert (Imdorf et al, 1994). Der Mittelwert der drei Luftproben ergab die durchschnittliche Luftkonzentration pro Exsikkator während der Behandlung. Dieser Wert wurde in den Abbildungen verwendet. Während der Behandlung lag die relative Lufffeuchtigkeit im Exsikkator zwischen 50 und $60 \%$.
Nach 72 Stunden wurden die getöteten Varroa und Bienen ausgezählt. Die überlebenden Varroa und Bienen wurden erfasst, indem die lebenden Bienen mit $\mathrm{CO}_{2}$ betäubt, in Alkohol ausgewaschen und ausgezählt wurden. Die Varroa- und Bienenmortalität entspricht dem Anteil der während der Behandlung getöteten Tiere an der Gesamtpopulation pro Exsikkator. Die Tests wurden 1992 und 1993 jeweils zwischen August und Mitte Oktober durchgeführt. Diese Periode wurde gewählt, da solche Substanzen nur zu diesem Zeitpunkt zur Bekämpfung der Varroa eingesetzt werden können. Zur Feststellung der Reproduzierbarkeit wurden vergleichbare Thymolkonzentrationen 1992 und 1993 getestet.

Die verwendeten ätherischen Substanzen von der Firma Fluka AG in Buchs waren Thymol ( $\mathrm{Nr}$ $89330,>99 \%)$, (t)-Campher ( $\mathrm{Nr} 21310, \sim 95 \%)$, Menthol ( $\mathrm{Nr} 63670,>98 \%)$ und Eucalyptol ( $\mathrm{Nr}$ 46090 , 99\%).

\section{ERGEBNISSE}

Insgesamt wurden 19 Kontrollbehandlungen (nur Frischluft) durchgeführt. Dabei wurde eine Bienenmortalität von $0,9 \%$ (SD \pm $1,3)$ und eine Varroa-Mortalität von $9,1 \%$ $(S D \pm 6,6)$ ermittelt.

Bei einer Thymolkonzentration von über $5 \mu \mathrm{g} / \mathrm{l}$ Luft lag die Varroa-Mortalität nach 72 Stunden bei $100 \%$ (Abb 1a). Die Bienenmortalität stieg bis $15 \mu \mathrm{g} / \mathrm{l}$ nur gering an. Bei höheren Werten nahm sie stark zu. Die vergleichbaren Thymolkonzentrationen, welche 1992 und 1993 getestet wurden, ergaben auch vergleichbare Werte. Sie sind in Abbildung 1a enthalten. Campher wies bereits bei $50 \mu \mathrm{g} / \mathrm{l}$ eine Varroa-Mortalität von nahezu 100\% auf (Abb 1b). Bis $150 \mu \mathrm{g} / \mathrm{l}$ war die Bienenverträglichkeit sehr gut. Bei Menthol nahm die Mortalität der Varroa zwischen 15 und $20 \mu \mathrm{g} / \mathrm{l}$ stark zu (Abb 1c). Ab $30 \mu \mathrm{g} / \mathrm{l}$ erreichte sie in den meisten Tests $100 \%$. Bis zu einer Konzentration von $60 \mu \mathrm{g} / \mathrm{l}$ lag die Bienenmortalität (mit einer Ausnahme) unter $10 \%$. Die Varroa-Mortalität stieg bei einer Eukalyptolkonzentrationen von $160 \mu \mathrm{g} / \mathrm{l}$ stark an (Abb 1d). Bei $240 \mu \mathrm{g} / \mathrm{l}$ 
a)

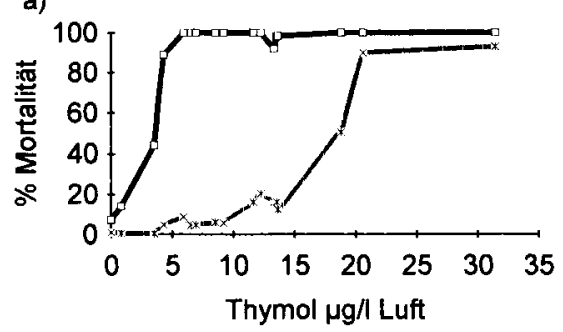

c)

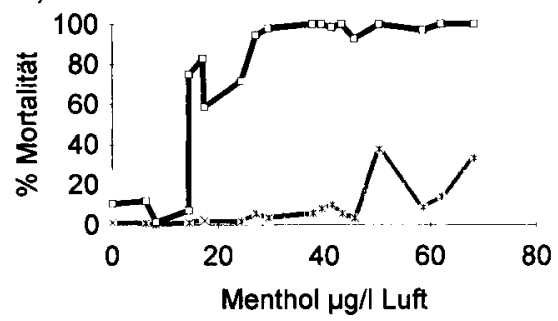

b)

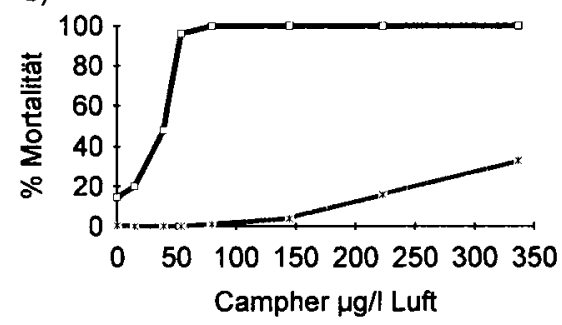

d)

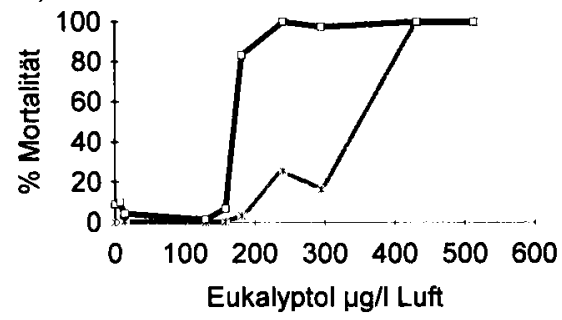

Abb 1. Mortalitätskurve für Varroa (*) und Bienen ( $\square$ ) nach 72 Stunden Behandlung mit unterschiedlichen Konzentrationen von Thymol (a), Campher (b), Menthol (c) und Eucalyptol (d).

erreichte sie $100 \%$. Gleichzeitig wurde bei dieser Konzentration eine Bienenmortalität von $25 \%$ ermittelt.

\section{DISKUSSION}

Neben Thymol, welches sich bereits in Form von 'Apilife VAR' als Wirkstoff zur VarroaBekämpfung in verschiedenen Kastentypen bewährt hat, scheinen auch Campher und Menthol die dazu nötigen Eigenschaften aufzuweisen. Praxisreife Formulierungen für die letzten beiden Substanzen liegen aber nicht vor. Mit Eucalyptol wurde bei einer Varroa-Mortalität von $100 \%$ bereits eine Bienenmortalität von $20-30 \%$ festgestellt. Deshalb, und wegen der schlecht kontrollierbaren Verdunstung bei Temperaturen um $30^{\circ} \mathrm{C}$, eignet sich reines Eucalyptol nicht als Varroa-Bekämpfungsmittel. Die bei einer Produktentwicklung zur Bekämpfung der Varroa anzustrebenden Konzentrationen in der Stockluft dürfte für Thymol, Cam- pher und Menthol bei 5-15, 50-150 resp 20-60 $\mu \mathrm{g} / \mathrm{l}$ liegen.

Während eines Screenings von ätherischen Ölen auf ihre varroazide Wirkung ermittelte Hoppe (1990) die Bienentoxizität nach 72 Stunden bei kleinen Gruppen von 20 gekäfigten Bienen in einem 3,4 I grossen Glasbehälter. Die Dosierung betrug ca $3 \mu \mathrm{l}$ ätherisches Öl pro I Luft. Für Thymian, Eukalyptus- und Pfefferminzöl wurde eine Bienentoxizität von 92,67 resp $48 \%$ ermittelt. Diese hohe Bienenmortalität dürfte auf eine Überdosierung zurückzuführen sein. Nach einer Untersuchung von Colin et al (1989) streut der Thymolgehalt von Thymianöl je nach Herkunft zwischen 5 und $40 \%$. Somit hätte im Test von Hoppe theoretisch eine Thymolkonzentration von ca 150 bis $1200 \mu \mathrm{g} / \mathrm{l}$ Luft entstehen können. In unserem Test wurde bereits bei $20 \mu \mathrm{g} / \mathrm{l}$ nach der gleichen Behandlungsdauer eine Bienenmortalität von $90 \%$ ermittelt.

Während der erfolgreichen Anwendung von 'Apilife VAR' im Schweizerkasten lag 
die Thymolkonzentration in der Stockluft im Brutnestbereich zwischen 3,6 und 7,5 $\mu \mathrm{g} / 1$ (Imdorf et al, 1994). Im Labortest wurden Konzentrationen von 5 bis $15 \mu \mathrm{g} / \mathrm{l}$ als optimal ermittelt. Dies zeigt, dass die Resultate des hier vorgestellten Labortests brauchbare Hinweise für die bei der Varroa-Bekämpfung anzustrebenden Konzentrationen von ätherischen Substanzen in der Stockluft geben. Mit Hilfe dieser Methode dürfte es auch möglich sein, die optimale Mentholkonzentration zur Bekämpfung von Acarapis woodi zu bestimmen.

Résumé - Effets toxiques du thymol, du camphre, du menthol et de l'eucalyptol sur Varroa jacobsoni Oud et Apis mellifera $L$ lors de tests en laboratoire. Afin de connaitre les effets varroacides et la tolérance de l'abeille aux huiles essentielles $(\mathrm{HE})$, le test en laboratoire suivant a été développé. Deux cages du type de Liebefeld contenant chacune 100 abeilles avec 20 à 40 varroas ont été placées dans un dessicateur et exposées à un courant d'air chargé en HE. Différentes concentrations ont été produites en mélangeant l'apport d'air frais, environ $500 \mathrm{ml} / \mathrm{min}$, avec des quantités différentes d'air chargé en HE (5 à $130 \mathrm{ml} / \mathrm{min}$, selon la substance testée et la concentration souhaitée) peu avant sa diffusion dans le dessicateur. Le traitement a été effectué en étuve à une température de $32^{\circ} \mathrm{C}$ et une humidité relative de 50 à $60 \%$. Après 24,48 et $72 \mathrm{~h}$, nous avons mesuré la concentration des composés actifs dans les dessicateurs. À cet effet, ce sont 1 à 20 I d'air qui, selon la substance active et la concentration, ont été aspirés au travers d'un tube d'absorption (Orbo-101, Supelco). La substance active a ensuite été extraite par du toluène et analysée par chromatographie en phase gazeuse. La valeur moyenne des 3 échantillons d'air a donné la concentration moyenne en $\mathrm{HE}$ pendant le traitement. L'un des 5 dessicateurs, alimenté avec de l'air frais non odo- risé $(550 \mathrm{ml} / \mathrm{min})$, a servi de contrôle. Après $72 \mathrm{~h}$, les varroas et les abeilles morts ont été dénombrés. Les abeilles survivantes ont été anesthésiées avec du $\mathrm{CO}_{2}$, lavées dans de l'alcool pour faire tomber les varroas restants, puis abeilles et varroas ont été comptés. La mortalité des varroas et des abeilles correspond au pourcentage d'individus tués pendant le traitement par rapport à la population totale. Les concentrations ayant tué près de $100 \%$ des varroas sans pour autant affecter les abeilles se situaient pour le thymol entre 5 et $15 \mu \mathrm{g} / \mathrm{l}$ (fig 1a), pour le camphre entre 50 et $150 \mu \mathrm{g} / \mathrm{l}$ (fig 1b) et pour le menthol entre 20 et $60 \mu \mathrm{g} / \mathrm{l}$ d'air (fig 1c). Quant à l'eucalyptol, on a constaté, pour une mortalité de varroas de $100 \%$, une mortalité des abeilles de $25 \%$ (fig 1d). Outre le thymol, qui a déjà fait ses preuves sous la forme $d^{\prime}$ '«Apilife VAR" dans différents types de ruches, le camphre et le menthol semblent posséder les caractéristiques d'un varroacide efficace. En revanche, l'eucalyptol $(240 \mu \mathrm{g} / \mathrm{l})$ ne convient pas en raison de son évaporation difficilement contrôlable et de ses effets toxiques affectant non seulement les varroas, mais aussi les abeilles.

Apis mellifera / Varroa jacobsoni / huile essentielle / toxicité / lutte chimique

\section{Summary - Toxic effects of thymol,} camphor, menthol and eucalyptol on Varroa jacobsoni Oud and Apis mellifera $L$ in a laboratory test. To study the toxic effects of volatile substances on Varroa and bees, the following test was developed. Two cages (Liebefeld type) with each 100 bees and 20 to 40 Varroa were placed in a desiccator and exposed to air contaminated with the volatile substances. Different air concentrations were produced by admixing fresh air $(500 \mathrm{ml} / \mathrm{min})$ to contaminated air (5-130 $\mathrm{ml} / \mathrm{min}$ depending on the active compound and the desired concentration). This treatment was performed in an incubator at 
a temperature of $32^{\circ} \mathrm{C}$ and 50 to $60 \%$ relative humidity. After 24,48 and $72 \mathrm{~h}$, the concentrations of the active compounds in the desiccators were measured. Depending on the active compound and the concentration, 1 to 20 l of air were sucked through adsorbent tubes (Orbo-10, Supelco). Thereafter, the active compounds were washed out with toluene and the extract analysed by gas chromatography. The values of the three air samples gave an average air concentration per treatment. One of 5 desiccators received fresh air only $(550 \mathrm{ml} / \mathrm{min})$ and served as a control. After $72 \mathrm{~h}$, dead bees and Varroa were counted. The living bees were numbed with $\mathrm{CO}_{2}$, washed in alcohol to seperate remaining Varroa from their hosts and the mites and bees counted. Varroa and bee mortality was expressed as the percentage of animals found dead during the treatment in the desiccator. The air concentration which killed nearly $100 \%$ of Varroa without noticeable loss of bees was found to be between 5 and $15 \mu \mathrm{g} / \mathrm{l}$ for thymol (fig 1a), between 50 and $150 \mu \mathrm{g} / \mathrm{l}$ for camphor (fig 1b) and between 20 and $60 \mu \mathrm{g} / \mathrm{l}$ air for menthol (fig 1c), $240 \mu \mathrm{g} /$ l eucalyptol produced $100 \%$ Varroa mortality but also $25 \%$ bee mortality (fig $1 \mathrm{~d}$ ). Thymol was found to be efficient as main compound of the varroacid "Apilife VAR" in different types of hives. Camphor and menthol also possess the necessary characteristics of an efficient varroacid. Eucalyptol however, is not very suitable for Varroa treatment since its rate of evaporation is difficult to control and only a small difference between its toxicity for Varroa and for bees was observed.

\section{Apis mellifera / Varroa jacobsoni / volatile oil / toxicity / chemical control}

\section{LITERATUR}

Chiesa $F$ (1991) Effective control of varroatosis using powdered thymol. Apidologie 22, 135-145

Colin M, Ducos de Lahitte J, Larribau, Boué T (1989) Activité des huiles essentielles de Labiées sur Asco- phaera apis et traitement d'un rucher. Apidologie 20, 221-228

Cox R, Wilson W, Maki D, Stoner A (1986) Chemical control of the honey bee tracheal mite Acarapis woodi. Am Bee J 131, 315-317

Cox R, Moffett J, Wilson W, Ellis M (1989) Effects of late spring and summer treatment on colony strenght, honey production and tracheal mite levels. Am Bee $J 129,547-549$

Delaplane K (1992) Controlling tracheal mites in colonies of honey bees with vegetable oil and menthol. $J$ Econ Entomol 85, 2118-2124

Duff S, Furgula B (1991) Some effects of menthol on honey bee tracheal mite infestations in nonmigratory honey bee colonies in Minesota. Am Bee $J 131$, 315-317

Duff S, Furgula B (1993) Evaluation of Amitraz and menthol as agents to control honey bee tracheal mite infestations in nonmigratory honey bee colonies in Minnesota. Am Bee $J 133,127-130$

Hoppe H (1990) Vergleichende Untersuchung zur biotechnischen Bekämpfung der Varroatose. Dissertation, Justus-Liebig-Universität, Giessen und Tierhygienisches Institut Freiburg, Deutschland

Liebig G (1993) Varroabekämpfung mit Apilife-VAR. Bienenpflege $9,247-249$

Mautz D (1982) Untersuchungen zur Bienengefährlichkeit von Thymol. Apidologie 13, 103-104

Moosbeckhoter R (1993) Versuche mit Apilife VAR zur Bekämpfung der Varroa-Milbe. Bienenwelt 7, 161166

Mutinelli F, Irsara A, Cremasco S, Piro R (1993) Utilizzo di Apilife-VAR sul vassoio di fondo per il controllo della varroasi. Apic Mod 84, 111-117

Imdorf A, Kilchenmann V, Maquelin C, Bogdanov S (1994) Optimierung der Anwendung von "Apilife VAR" zur Bekämpfung von Varroa jacobsoni Oud in Bienenvölkern. Apidologie 25, 49-60

Rickli M, Imdorf A, Kilchenmann V (1991) VarroaBekämpfung mit Komponenten von ätherischen Ölen. Apidologie 22, 417-421

Schulz S (1993) Anwendung thymolhaltiger Varroazide bei Magazinvölkern. Dtsches Bienen J 8, 18-20

Van der Steen J (1992) Der Effekt einer Mischung ätherischer Öle auf die Varroa-Infektion in Bienenvölkern. Apidologie 23, 383-385

Vecchi M und Giordani G (1968) Chemotherapy of acarine disease. 1. Laboratory test. $J$ Invertebr Pathol $10,390-416$

Vorwohl G, Liebig G, Rüdiger G, Maier M (1994) Bericht der Landesanstalt für Bienenkunde der Universität Hohenheim für das Jahr 1993. Bienenpflege 3, 67-82

Wilson W, Cox R, Moffett J, Ellis M (1990) Improved survival of honey bee colonies from long term supression of tracheal mites Acarapis woodi with menthol. BeeScience 1, 48-54 